\title{
Optimal Spacing Policy for Vehicle Platoon Control with Road-Friction Coefficient
}

\author{
Lei Zuo $\mathbb{D}$, Duo Meng, and Jinqi Zhang \\ Department of Electrical and Control Engineering, Chang'an University, Xi'an, China \\ Correspondence should be addressed to Lei Zuo; 1_zuo@chd.edu.cn
}

Received 15 July 2021; Revised 17 November 2021; Accepted 26 November 2021; Published 30 December 2021

Academic Editor: Zhihong Yao

Copyright (c) 2021 Lei Zuo et al. This is an open access article distributed under the Creative Commons Attribution License, which permits unrestricted use, distribution, and reproduction in any medium, provided the original work is properly cited.

\begin{abstract}
This paper investigates the vehicle platoon control problems, in which the road-friction coefficient is taken into consideration. In order to improve the vehicle platoon safety in various road-friction conditions, an optimal spacing policy is proposed for the vehicle platoon. In detail, an intervehicle space optimization framework is developed by using a safety cost function and the gradient decent method. In this way, the optimal intervehicle spacing headway is presented such that the vehicle can be safely driven to the desired platoon under various road-friction conditions. Then, based on the proposed optimal spacing policy, we transform this optimal spacing vehicle platoon control problem into a moving target tracking problem. An adaptive distributed integrated sliding mode (DISM)-based vehicle platoon control scheme is proposed such that the vehicles can effectively follow the presented optimal spacing platoon. Moreover, the stability of the proposed vehicle platoon system is strictly analyzed and numerical simulations are provided to verify the proposed approaches.
\end{abstract}

\section{Introduction}

Recently, increasing attention has been paid to the vehicle platoon control due to its significant benefits on traffic safety, mobility, and environment [1-4]. It can substantially improve the intelligent transportation system by emerging the multiagent control techniques and the traffic transportation theories. The main objective of vehicle platoon control is to automatically drive all vehicles moving along the given line with desired intervehicle space and a certain velocity. There are several important parameters involved in vehicle platoon control, such as the platoon control of nonlinear vehicles [5], platoon size optimization [6], and platoon spacing policy [7].

Usually, the platoon spacing policy presents a strategy to adjust the intervehicle spacing/time headway for a vehicle platoon. It has a great impact on traffic, especially in reducing the fuel consumption and improving the traffic safety. The fuel consumption would be reduced when the intervehicle space is small. However, the small intervehicle space will affect the safety of the entire vehicle platoon [8]. To fill this gap, many researchers pay much attention on the vehicle platoon control with various spacing policies [9-15].
In general, the spacing policy for vehicle platoon can be classified into two cases: the constant spacing policy (CSP) and the varying spacing policy (VSP). In the vehicle platoon with CSP, a platoon control strategy is usually proposed to adjust the intervehicle spacing headway into a constant. It is a basic vehicle platoon control strategy and has been widely investigated. For instance, a distributed model predictive control scheme is proposed to maintain the desired intervehicle space in [16]. In [17], the vehicle platoon control with different communication topologies is studied, where the constant spacing policy is employed. In [7], a vehicle platoon control method with CPS is proposed, where the communication delay is taken into consideration. An adaptive sliding mode-based platoon control method with both prescribed tracking performance and constant spacing policy is proposed in [18], where the actuator saturation, uncertain parameters, and unknown disturbances are all considered. A two-layer decentralized and adaptive control scheme for heterogeneous connected vehicle platoon is designed to ensure equal and constant intervehicular spacing in [19]. In [20], the control problem for leader-following (LF) vehicle platoons with a constant spacing policy is 
studied, where the information of the leader is broadcasted to each follower subject to varied communication ranges. In [21], a cooperative optimal power split (COPS) method for a group of intelligent electric vehicles (EVs) traveling on a highway with varying slopes is proposed, which aims to extend the battery lifespan and reduce energy consumption. In [22], a highway-based ecological cooperative adaptive cruise control (Eco-CACC) strategy with constant spacing policy is proposed for a heterogeneous platoon of heavyduty vehicles, where the time delays are taken into consideration.

For the vehicle platoon control with VSP, there are several classical spacing policy, such as constant time headway spacing policy, the quadratic spacing policy, and the adaptive spacing policy. For instance, the vehicle platoon control algorithm with modified constant time headway spacing policy is proposed in [23], where the nonzero initial spacing errors' case is taken into consideration. A novel vehicle platoon control scheme with quadratic spacing policy is proposed in [24], where the traffic flow stability and energy consumption are considered. While considering the impact of different control architecture on spacing strategies, a control method with an adaptive spacing policy for multibrand ad hoc platoon is presented in [25]. In [3], an improved quadratic spacing policy with the lower bound of fault factor is proposed, and the fault-tolerant control problem for heterogeneous vehicular platoons is investigated where input quantization and dead-zone nonlinearity are also involved. A constant time headway policy-based distributed integral sliding mode (ISM) control strategy is proposed in [26], which focuses on the car-following interactions between vehicles. Meanwhile, in [27], a variable structure control approach for vehicles' platooning based on a hierarchical fuzzy logic is proposed by employing two common spacing policies: constant distance and constant time headway. A new spacing policy combining constant distance policy (CDP) and constant time headway policy (CTHP) schemes are proposed to achieve string stability and improve the road capacity with consideration of unknown parameters, disturbances and actuator saturation, in [28]. In [29], a switched control strategy of heterogeneous vehicle platoon for multiple objectives with state constraints is proposed, in which the four major objectives including vehicle safety, passenger comfort, formation control, and fuel economy are all taken into account. Thus, to achieve the above four objectives, the distributed model predictive control with multiple objectives (DMPCMO) is designed.

Besides, there are still some literatures which apply the platoon spacing policy on other fields. In [30], a tracking controller of multiple autonomous cars or car-like robots adapts the leader-follower strategy and the prescribed performance technique is designed without any collision and singularity. A platoon controller is proposed with constrained relative range and angles in [31], which mainly studies the three-dimensional platoon control of multiple underactuated autonomous underwater vehicles (AUVs) subjected to environmental disturbances and model uncertainties. A robust platoon controller for a convoy of tractor-trailer wheeled mobile robots (TTWMRs) with a guaranteed prescribed performance under the collision avoidance and connectivity maintenance is successfully proposed based on only posture measurements in [32].

Although the vehicle platoon with particular spacing policy has been well studied, the current presented spacing policies are only related to the vehicle's position and velocity. There are few results taking the road conditions (such as snow, rain, and fog) into consideration. In fact, it is inevitable that the vehicle platoon is driven in the snow or the rain. In that case, the safety of vehicle platoon is greatly affected, and it might lead to some serious traffic accident. Hence, it is necessary to specifically study the vehicle platoon control in such scenarios. Essentially, the relationship between the road conditions and the vehicle dynamics can be illustrated by the road-friction coefficient. Different weathers around the road, including the snow and rain, give us different road frictions. When there is snow on the road, the road-friction coefficient becomes small, and we prefer to increase the intervehicle space to improve the platoon safety. On this basis, it is necessary to further investigate the optimal spacing policy in vehicle platoon control with different road-friction coefficients.

Motivated by this fact, an optimal spacing policy for the vehicle platoon with different road-friction coefficients is proposed. Then, a distributed integrated sliding mode (DISM)-based platoon control strategy is presented such that the vehicle can strictly follow the desired spacing policy. Different from the previous spacing policy, the optimal spacing policy in this paper is dynamic, and the optimal spacing values are different under varying road-friction coefficients. The main contributions are twofold:

(i) An optimal spacing policy is proposed for the vehicle platoon with various road frictions. In detail, a safety cost function is presented to describe the safety performance of the vehicle platoon with different road-friction coefficient. Then, an intervehicle space optimization framework is developed to find out the optimal spacing policy. Compared with the previous constant spacing policy, this framework fully combines the vehicle platoon control and the road conditions, and it would greatly improve vehicle platoon safety and is much more consistent with the actual situation at the same time.

(ii) Based on the proposed optimal spacing policy, a novel vehicle platoon control scheme is proposed by using the DISM technique. The external disturbances in the vehicle dynamics are taken into consideration, and a novel disturbance estimation method is developed for the platoon control scheme. Moreover, the stability and feasibility of the proposed vehicle platoon control system with optimal spacing policy are strictly analyzed.

The remainders of this paper are presented as follows. In Section 2, the preliminaries and problem formulation are presented. Then, an optimal spacing policy and the DISMbased vehicle platoon control scheme are provided in Section 3 and Section 4, respectively. To illustrate the proposed 
approaches, numerical simulations are presented in Section 5. In final, Section 6 draws the conclusion.

\section{Preliminaries and Problem Formulation}

Consider a group of vehicles randomly on a given line $\mathscr{L}$. The dynamic model of each vehicle is described by

$$
\left\{\begin{array}{l}
\dot{p}_{i}(t)=v_{i}(t) \\
\dot{v}_{i}(t)=u_{i}(t)+\delta_{i}(t), i=1, \ldots, n
\end{array},\right.
$$

where $v_{i}(t)$ and $p_{i}(t)$ are the velocity and position of the $i$ th vehicle, respectively, $u_{i}(t)$ is the control input of the $i$ th vehicle, and $\delta_{i}(t)$ is unknown external disturbance of the $i$ th vehicle satisfying that $\underline{\delta}_{i}(t) \leq \delta_{i}(t) \leq \bar{\delta}_{i}(t)$.

Suppose that each vehicle has the capability of communication, computing, and sampling. Then, we define the communication topology among vehicles as $\mathscr{G}$. Each vehicle is denoted as the node, and there would be an edge if any two vehicles can communicate with each other. The detailed communication topology is illustrated in Figure 1.

To quantitatively describe the platoon safety on the given road, a safety-cost function will be provided involving both the vehicle platoon and the road friction. Particularly, for the road-friction coefficient, since the road is usually stretched across many regions, the road-friction coefficient is varying associated with the locations. Hence, a friction function in terms of the locations must be provided to fully describe the road-friction coefficient. Moreover, the distance to the vehicle is also an importance parameter to evaluate the platoon safety (see Figure 1). Hence, a safety-cost function is presented as follows:

$$
H(P)=\sum_{i=1}^{n} \int_{W_{i}}\left\|p_{i}-q\right\|^{2} \phi(q) d q,
$$

where $\mathscr{L}$ is the given road and $P=\left[p_{1}, \ldots, p_{n}\right]^{T}$ is the position vector, $W_{i}$ denotes the safety-cost region to the $i$ th vehicle, $\phi(q): \mathscr{L} \longrightarrow R^{+}$denotes the road-friction function over the road, and $q \in \mathscr{L}$ is an arbitrary point on $\mathscr{L}$.

On this basis, the objective of this paper is that we will find the optimal vehicle platoon spacing policy associated with the road-friction coefficient. Then, based on the DISM, a novel vehicle platoon control scheme will be proposed to follow the desired vehicle platoon.

\section{Optimal Spacing Policy}

It is noticed that there are two variables in (2) affecting the platoon safety performance. The assigned region partition $W_{i}$ and the vehicle's position $p_{i}$. To optimize this safety-cost function in (2), we should firstly clarify the region partition strategy. Then, the following lemma is provided [33].

Lemma 1. Suppose there are $n$ vehicles in a bounded line. The optimal disjointed partition strategy for these vehicles is the Voronoi partition, which is shown as

$$
V_{i}=\left\{q \in \mathscr{L}\left\|p_{i}-q\right\| \leq\left\|p_{j}-q\right\|, \forall i, j \in n, j \neq i\right\},
$$

where $V_{i}$ is called the ith agent's Voronoi region.

Then, the safety-cost function in (2) becomes

$$
H(P, \mathscr{V})=\sum_{i=1}^{n} \int_{V_{i}}\left\|p_{i}-q\right\|^{2} \phi(q) d q,
$$

where $\mathscr{V}=\left\{V_{1}, \ldots, V_{n}\right\}$.

To minimize the function $H(P, \mathscr{V})$, we need to find the optimal position $p_{i}^{*}$ for each vehicle. According to the optimization methods, the safety-cost function $H(P, \mathscr{V})$ will be minimized when $p_{i}$ satisfies that

$$
\frac{\partial H}{\partial p_{i}}=M_{V_{i}}\left(p_{i}^{*}-C_{V_{i}}\right)=0, i=1, \ldots, n .
$$

where $M_{V_{i}}$ and $C_{V_{i}}$ are the mass and centroid point of $V_{i}$, which are shown by

$$
M_{V_{i}}=\int_{V_{i}} \phi(q) d q, C_{V_{i}}=\int_{V_{i}} q \phi(q) d q / \int_{V_{i}} \phi(q) d q .
$$

On this basis, we obtain that

$$
p_{i}^{*}=C_{V_{i}}, i=1, \ldots, n \text {. }
$$

Note that the centroid $C_{V_{i}}$ of Voronoi region is the desired vehicle platoon position for the $i$ th vehicle with the road-friction function $\phi(q)$. Then, we can transform this optimal spacing vehicle platoon control problem into a target tracking one, in which the centroid $C_{V_{i}}$ is the target point. In detail, we define the tracking error $e_{i}(t)$ as follows:

$$
e_{i}(t)=\bar{p}_{i}^{*}-p_{i}(t)
$$

Moreover, we define a virtual leader vehicle and a virtual tail vehicle to limit the safety-cost region of the entire vehicle platoon. The dynamics of these virtual leader and tail vehicles are described by

$$
\left\{\begin{array}{l}
\dot{p}_{l}(t)=v_{l}(t) \\
\dot{p}_{0}(t)=v_{0}(t)
\end{array},\right.
$$

where $p_{l}(t)$ and $v_{l}(t)$ are the position and the velocity of the virtual leader vehicle and $p_{0}(t)$ and $v_{0}(t)$ are for the virtual tail vehicle.

\section{The Adaptive DISM-Based Vehicle Platoon Control}

Based on the above analyses, it shows that the optimal position for each vehicle is its centroid in the Voronoi region. When we only focus on one vehicle tracking its Voronoi centroid, the vehicle platoon control with optimal spacing policy can be transformed into a target tracking problem. Inspired by this idea, a novel adaptive DISM-based vehicle platoon control scheme is proposed for a group of vehicles in this section.

Firstly, the integrated sliding mode surface for the $i$ th vehicle is shown as

$$
s_{i}(t)=\dot{e}_{i}(t)+\alpha_{1} e_{i}(t)+\alpha_{2} \int_{0}^{t} e_{i}(\tau) \mathrm{d} \tau
$$


where $\alpha_{1}$ and $\alpha_{2}$ are positive constants.

In order to guarantee the stability of vehicle platoon system, an improved coupled sliding surface is provided as follows:

$$
S_{i}(t)=\left\{\begin{array}{ll}
q s_{i}(t)-s_{i+1}(t), & i=1, \ldots, n-1 \\
q s_{i}(t), & i=n
\end{array},\right.
$$

where $q \neq 0$ is a positive constant parameter. The relationship between $S_{i}(t)$ and $s_{i}(t)$ is shown as

$$
S(t)=Q s(t),
$$

where $s(t)=\left[s_{1}(t), \ldots, s_{n}(t)\right]^{T}, s(t)=\left[s_{1}(t), \ldots, s_{n}(t)\right]^{T}$, and

$$
\begin{aligned}
\dot{S}_{i}(t) & =q \dot{s}_{i}(t)-\dot{s}_{i+1}(t) i=1, \ldots, n-1 \\
& =q\left(\ddot{e}_{i}(t)+\alpha_{1} \dot{e}_{i}(t)+\alpha_{2} e_{i}(t)\right)-\left(\ddot{e}_{i+1}(t)+\alpha_{1} \dot{e}_{i+1}(t)+\alpha_{2} e_{i+1}(t)\right) \\
& =q\left(\ddot{p}_{i-1}(t)-u_{i}(t)-\delta_{i}(t)+\alpha_{1} \dot{e}_{i}(t)+\alpha_{2} e_{i}(t)\right)-\left(u_{i}(t)+\delta_{i}(t)-\ddot{p}_{i+1}(t)+\alpha_{1} \dot{e}_{i+1}(t)+\alpha_{2} e_{i+1}(t)\right) \\
& =-(q+1)\left(u_{i}(t)+\delta_{i}(t)\right)+D_{i}(t),
\end{aligned}
$$

$$
Q=\left[\begin{array}{ccccc}
Q & -1 & \cdots & 0 & 0 \\
0 & Q & -1 & \cdots & 0 \\
\vdots & \vdots & \ddots & \vdots & \vdots \\
0 & 0 & \cdots & Q & -1 \\
0 & 0 & \cdots & 0 & Q
\end{array}\right] .
$$

Since $q \neq 0$ and $Q$ is invertible, when $S_{i}(t)$ is zero, $S_{i}(t)$ becomes zero at the same time. Then, according to (1), (7), and (8), $\dot{S}_{i}$ becomes where $\quad D_{i}(t)=q \ddot{p}_{i-1}(t)+\ddot{p}_{i+1} \quad(t)+\alpha_{1}\left(q \dot{e}_{i}(t)-\dot{e}_{i+1}(t)\right)+$ $\alpha_{2}\left(q e_{i}(t)-e_{i+1}(t)\right)$.

For the case $i=n$, we obtain that

$$
\begin{aligned}
\dot{S}_{n}(t) & =q \dot{s}_{n}(t) \\
& =q\left[\ddot{e}_{n}(t)+\alpha_{1} \dot{e}_{n}(t)+\alpha_{2} e_{n}(t)\right] \\
& =-q\left(u_{n}(t)+\delta_{n}(t)\right)+D_{n}(t),
\end{aligned}
$$

where $D_{n}(t)=q\left(\ddot{p}_{n-1}(t)+\alpha_{1} \dot{e}_{n}(t)+\alpha_{2} e_{n}(t)\right)$.

Then, based on the boundaries of external disturbances, we have that

$$
\begin{aligned}
& \left|\tilde{\bar{\delta}}_{i}(t)=\widehat{\bar{\delta}}_{i}(t)-\bar{\delta}_{i}(t)\right| \leq \widetilde{\bar{\delta}}_{\text {imax }}, \\
& \left|\widetilde{\delta}_{i}(t)=\underline{\widehat{\delta}}_{i}(t)-\underline{\delta}_{i}(t)\right| \leq \widetilde{\widetilde{\delta}}_{\text {max }},
\end{aligned}
$$

where $\overline{\bar{\delta}}_{i}(t)$ and $\underline{\widehat{\delta}}_{i}(t)$ are the estimation of $\bar{\delta}_{i}(t)$ and $\underline{\delta}_{i}(t)$, respectively, and $\widetilde{\bar{\delta}}_{i \max }$ and $\underline{\tilde{\delta}}_{\text {max }}$ are the maximum estimation errors.

In addition, the following lemma is provided to show the stability of the proposed vehicle platoon systems [34].

Lemma 2. If $\Delta(t): R \longrightarrow R^{+}$is a uniformly continuous function for $t \geq 0$ and the limit of the integral $\lim _{t \rightarrow \infty} \int_{0}^{t} \Delta(\tau) d(\tau)$ exists, then it satisfies that

$$
\lim _{t \rightarrow \infty} \Delta(t)=0 .
$$

On this basis, we have the following adaptive DISMbased vehicle platoon control schemes:

$$
\begin{aligned}
& u_{i}(t)=\frac{1}{q+1}\left(D_{i}(t)+k \operatorname{sgn}\left(S_{i}(t)\right)\right)-\left[\left(1-\mu_{i}\right) \hat{\bar{\delta}}_{i}(t)+\mu_{i} \widehat{\widehat{\delta}}_{i}(t)\right], i=1,2, \ldots, n-1 ; \\
& u_{n}(t)=\frac{1}{q}\left(D_{i}(t)+k \operatorname{sgn}\left(S_{i}(t)\right)\right)-\left[\left(1-\mu_{i}\right) \hat{\bar{\delta}}_{i}(t)+\mu_{i} \widehat{\hat{\delta}}_{i}(t)\right], i=n,
\end{aligned}
$$

where

$$
D_{i}(t)=\left\{q \ddot{p}_{i-1}(t)+\ddot{p}_{i+1}(t)+\alpha_{1}\left(q \dot{q}_{i}(t)-\dot{e}_{i+1}(t)\right)+\alpha_{2}\left(q e_{i}(t)-e_{i+1}(t)\right), i=1, \ldots, n-1 q\left(\ddot{p}_{n-1}(t)+\alpha_{1} \dot{e}_{n}(t)+\alpha_{2} e_{n}(t)\right), i=n,\right.
$$

where $k$ is the positive design parameter of the controller satisfying that

$$
k>|q| \max _{1 \leq i \leq n}\left\{\widetilde{\bar{\delta}}_{\max i}, \underline{\tilde{\delta}}_{\max i}\right\} .
$$



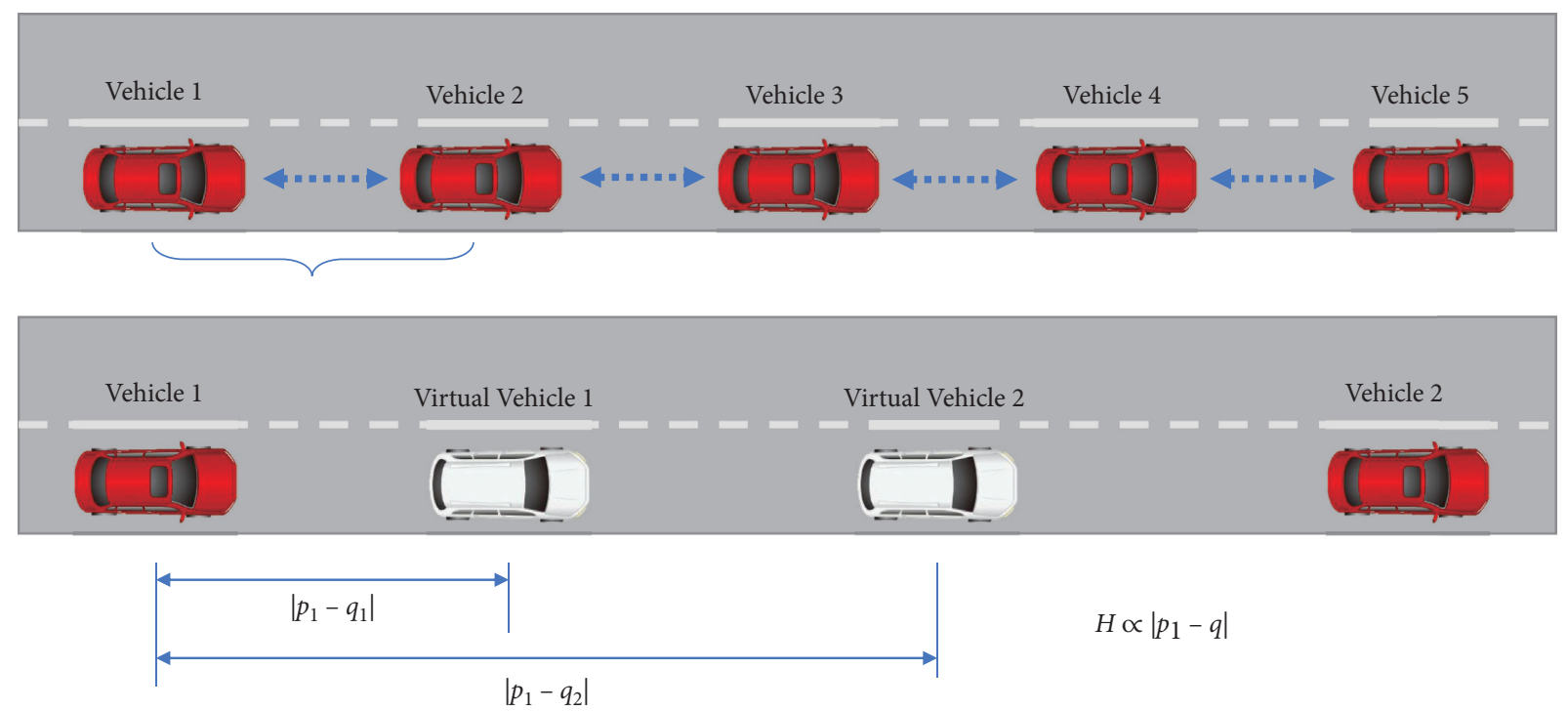

Figure 1: The vehicle platoon communication and safety-cost function.

The adaptive laws for $\widehat{\bar{\delta}}_{i}(t)$ and $\underline{\hat{\delta}}_{i}(t)$ are given as

$$
\dot{\overline{\widehat{\delta}}}_{i}(t)=\underline{\widehat{\hat{\delta}}}_{i}(t)=-(q+1) \gamma_{i} S_{i}(t), i=1, \ldots, n-1, \dot{\overline{\widehat{\delta}}}_{n}(t)=\underline{\widehat{\hat{\delta}}}_{n}(t)=-\gamma_{i} q S_{i}(t), i=n,
$$

where $\gamma_{i}$ is a positive constant and $\mu_{i}$ is denoted as

$$
\mu_{i}=\left\{\begin{array}{cc}
1, & q>0, S_{i}(t)>0 \\
0, & q>0, S_{i}(t) \leq 0 \\
1, & q \leq 0, S_{i}(t) \leq 0 \\
0, & q \leq 0, S_{i}(t)>0
\end{array}\right.
$$

To formally present the tracking behaviors of vehicles and strictly show the stability of proposed control system, a theorem is provided as follows.

Theorem 1. Consider a group of vehicles on a given line $\mathscr{L}$ described by (1). The road-friction coefficient over this line is denoted by $\phi(q)$ and the optimal spacing policy is presented in (6). Then, by using the vehicle platoon control scheme in (14), the vehicles will be driven to the desired vehicle platoon with the desired intervehicle spacing headway.
Proof. Consider the following Lyapunov function candidate:

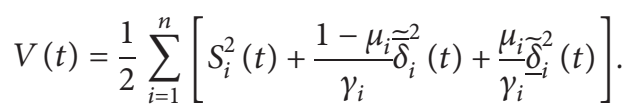

Ass $\bar{\delta}_{i}$ and $\underline{\delta}_{i}$ are constants, it follows that $\dot{\overline{\bar{\delta}}}_{i}(t)=\dot{\overline{\bar{\delta}}}_{i}(t)$ and $\underline{\delta}_{i}(t)=\underline{\hat{\delta}}_{i}(t)$. Then, taking the derivation of $V$, we obtain that

$$
\dot{V}(t)=\sum_{i=1}^{n}\left[S_{i}(t) \dot{S}_{i}(t)+\frac{1-\mu_{i}}{\gamma_{i}} \widetilde{\bar{\delta}}_{i}(t) \dot{\overline{\hat{\delta}}}_{i}(t)+\frac{\mu_{i}}{\gamma_{i}} \underline{\hat{\delta}}_{i}(t) \underline{\hat{\delta}}_{i}(t)\right] .
$$

Substituting (11) and (12) into $\dot{V}$, it follows

$$
\begin{aligned}
& \dot{V}_{i}(t)=S_{i}(t)\left[-(q+1)\left(u_{i}(t)+\delta_{i}(t)\right)+D_{i}(t)\right]+\frac{1-\mu_{i}}{\gamma_{i}} \widetilde{\bar{\delta}}_{i}(t) \dot{\overline{\hat{\delta}}}_{i}(t)+\frac{\mu_{i}}{\gamma_{i}} \widetilde{\delta}_{i}(t) \underline{\hat{\widehat{\delta}}}_{i}(t), i=1, \ldots, n-1, \\
& \dot{V}_{n}(t)=S_{i}(t)\left[-q\left(u_{i}(t)+\delta_{i}(t)\right)+D_{i}(t)\right]+\frac{1-\mu_{i}}{\gamma_{i}} \widetilde{\bar{\delta}}_{i}(t) \dot{\overline{\hat{\delta}}}_{i}(t)+\frac{\mu_{i}}{\gamma_{i}} \widetilde{\delta}_{i}(t) \dot{\hat{\widehat{\delta}}}_{i}(t), i=n,
\end{aligned}
$$

where $V_{i}$ is the $i$ th element in $V$ and $D_{i}(t)$ is presented in (19).

According to the definition of $\mu_{i}$ in (22), we have that 
$\left.\begin{array}{l}-(q+1) \delta_{i}(t) S_{i}(t) \leq-(q+1)\left[\left(1-\mu_{i}\right) \bar{\delta}_{i}(t)+\mu_{i} \underline{\delta}_{i}(t)\right] S_{i}(t) \\ -q \delta_{i}(t) S_{i}(t) \leq-q\left[\left(1-\mu_{i}\right) \bar{\delta}_{i}(t)+\mu_{i} \underline{\delta}_{i}(t)\right] S_{i}(t)\end{array}\right]$.
Then, substituting (14) into (20), it follows that

$$
\begin{aligned}
& \left.\dot{V}_{i}(t)=S_{i}(t)\left[(q+1)\left[\left(1-\mu_{i}\right) \overline{\bar{\delta}}_{i}(t)+\mu_{i} \widehat{\underline{\delta}}_{i}(t)\right]\right]-S_{i}(t) \operatorname{ksgn}\left(S_{i}(t)\right)-S_{i}(t)(q+1) \delta_{i}(t)\right)+\frac{1-\mu_{i}}{\gamma_{i}} \widetilde{\bar{\delta}}_{i}(t) \dot{\overline{\hat{\delta}}}_{i}(t)+\frac{\mu_{i}}{\gamma_{i}} \widetilde{\delta}_{i}(t) \dot{\hat{\delta}}_{i}(t) \\
& =(q+1)\left[\left(1-\mu_{i}\right) \overline{\bar{\delta}}_{i}(t)+\mu_{i} \widehat{\hat{\delta}}_{i}(t)\right] S_{i}(t)-k\left|S_{i}(t)\right|-(q+1) \delta_{i}(t) S_{i}(t)+\frac{1-\mu_{i}}{\gamma_{i}} \widetilde{\bar{\delta}}_{i}(t) \dot{\bar{\delta}}_{i}(t)+\frac{\mu_{i}}{\gamma_{i}} \widetilde{\delta}_{i}(t) \dot{\hat{\bar{\delta}}}_{i}(t) \\
& \leq k\left|S_{i}(t)\right|+(q+1)\left[\left(1-\mu_{i}\right) \hat{\bar{\delta}}_{i}(t)+\mu_{i} \underline{\hat{\delta}}_{i}(t)\right] S_{i}(t)+\frac{1-\mu_{i}}{\gamma_{i}} \tilde{\bar{\delta}}_{i}(t) \dot{\overline{\hat{\delta}}}_{i}(t)+\frac{\mu_{i}}{\gamma_{i}} \widetilde{\delta}_{i}(t) \dot{\hat{\delta}}_{i}(t)=-k\left|S_{i}(t)\right| \leq 0 .
\end{aligned}
$$

For the case $i=n$, we have that $\dot{V}_{n}(t) \leq-k\left|S_{n}(t)\right| \leq 0$. Then, the above equation can be rewritten as

$$
\dot{V}_{i}(t) \leq-k \sum_{i=1}^{n}\left|S_{i}(t)\right|=-\Delta(t) \leq 0,
$$

where $\Delta(t) \triangleq k \sum_{i=1}^{n}\left|S_{i}(t)\right| \geq 0$. On this basis, we have that

$$
V_{i}(0)-V_{i}(t) \geq \int_{0}^{t} \Delta(\tau) \mathrm{d} \tau .
$$

Recalling that $\dot{V}_{i}(t) \leq 0$ and $V_{i}, \widetilde{\bar{\delta}}_{\dot{q}}(t)$, and $\tilde{\delta}_{i}(t)$ are all bounded, it indicates that $\lim _{t \rightarrow \infty} \int_{0}^{\dot{t}} \Delta(\tau) d \tau$ is bounded. Then, according to Lemma 2, we have that $\lim _{t \rightarrow \infty} \Delta(t)=\lim _{t \rightarrow \infty} k \sum_{i=1}^{n}\left|S_{i}(t)=0\right|$. Since $k$ is positive and $\lim _{t \rightarrow \infty} k \sum_{i=1}^{n}\left|S_{i}(t)=0\right|$, we obtain that $s_{i}(t)$ and $e_{i}(t)$ will converge to zeros, which indicates the stability of our proposed vehicle platoon control system. Namely, the vehicles on the given line will converge to the desired platoon. This ends the proof.
To solve the chattering problem caused by $\operatorname{sgn}\left(S_{i}(t)\right)$, the function $S_{i}(t) /\left|S_{i}(t)\right|+\sigma$ will be employed, where $\sigma$ is a small enough positive constant. In addition, the indicator function $\mu_{i}(t)$ may also cause chattering in practical application. Hence, a smooth continuous function is introduced as follows:

$$
\bar{\mu}_{i}(t)=\left\{\begin{array}{ll}
\frac{1}{1+e^{-a\left(S_{i}(t)-b\right)},} & \text { if } q>0 \\
\frac{1}{1+e^{a\left(S_{i}(t)+b\right)},}, & \text { if } q<0
\end{array},\right.
$$

where $a \gg 1$ and $0<b \ll 1$.

On this basis, an improved platoon control scheme can be rewritten as

$$
\begin{aligned}
& u_{i}^{*}(t)=-\left[\left(1-\bar{\mu}_{i}(t)\right) \hat{\bar{\delta}}_{i}(t)+\bar{\mu}_{i}(t) \widehat{\widehat{\delta}}_{i}(t)\right]+\frac{D_{i}(t)}{q+1}+\frac{k}{q+1} \frac{S_{i}(t)}{\left|S_{i}(t)\right|+\sigma}, i=1,2, \ldots, n-1, \\
& u_{n}^{*}(t)=-\left[\left(1-\bar{\mu}_{i}(t)\right) \hat{\bar{\delta}}_{i}(t)+\bar{\mu}_{i}(t) \widehat{\hat{\delta}}_{i}(t)\right]+\frac{1}{q}\left(D_{i}(t)+k \frac{S_{i}(t)}{\left|S_{i}(t)\right|+\sigma}\right), i=n .
\end{aligned}
$$

Based on the above theorem and analyses, the following algorithm is provided to illustrate the procedure of the proposed vehicle platoon control system.

While proceeding the vehicle platoon control, it is worth to note that the optimal spacing policy is varying in associated with the vehicle positions. It is because the Voronoi partition is based on the vehicles' current positions [33]. Once the vehicles update their positions, the Voronoi partition is also changed. It will lead the optimal position of the vehicle to another point. The final stable state of the vehicle platoon is that all the vehicles reach their own optimal positions and have the same velocity. Moreover, in order to effectively drive the vehicles to their optimal positions, the control gain $k$ should be chosen large enough while ensuring the stability of the proposed vehicle platoon system [34] (Algorithm 1).

\section{Numerical Simulations}

Consider 9 vehicles in a given line $\mathscr{L}$. The dynamics of each vehicle are described by (1). The road-friction coefficient on $\mathscr{L}$ is shown by

$$
\phi(q)=2000 \sin \left(\frac{\pi}{400}(q-200)\right)+2000 .
$$


Input: the initial position: $P(0)=\left[p_{1}, \ldots, p_{n}\right]^{T}$; the initial velocity: $V(0)=\left[v_{1}, \ldots, v_{n}\right]^{T}$; the road-friction coefficient function: $\phi(q)$; the controller parameters including $k, q, \gamma_{i}, \alpha_{1}, \alpha_{2}, \delta, a, b$;

Output: the vehicle platoon with optimal spacing policy.

(1) According to (5), calculate the centroids of Voronoi regions $C_{V_{i}}$ with initial positions $P(0)$;

(2) for $t=t_{0}: \Delta t: t_{f}$ do

(3) for $i=1, \ldots, n$ do

(4) According to (7), calculate $e_{i}(t)$;

(5) Calculate $\dot{e}_{i}(t)$ and $\ddot{e}_{i}(t)$ based on (1);

(6) Construct integrated sliding surface $S_{i}(t)$ by employing (8);

(7) Based on (11) and (19), Calculate the $S_{i}(t)$ and $D_{i}(t)$;

(8) According to (23), calculate $u_{i}(t)$;

(9) According to (1), update the positions of vehicles, $p_{i}(t+1)$;

(10) end for

(11) Let $P(t+1)=\left[p_{1}(t+1), \ldots, p_{n}(t+1)\right]^{T}$. Then, calculate the updated Voronoi region $V_{i}(t+1)$ and centroid $C_{V_{i}}(t+1)$;

(12) end for

Algorithm 1: The vehicle platoon control algorithm with optimal spacing policy.

where $q$ is an arbitrary point on $\mathscr{L}$. The details are presented in Figure 2.

The evolution of road-friction coefficient on $\mathscr{L}$ is provided in Figure 2. Notice that the value of $\phi(q)$ is close to zero around the time $150 s$ and $300 s$ in finite simulation time. This theoretical optimal spacing should be increased for safety of the platoon.

The initial position of the virtual leader is set as $p_{0}(0)=$ $100 \mathrm{~m}$ and $v_{0}(0)=5 \mathrm{~m} / \mathrm{s}$. The initial positions of vehicles are randomly located on the given line, and the initial velocities of the vehicles are set as $v(0)=[1,1,1,1,1,1,1,1,1]$. The external disturbances $\delta_{i}(t)$ are presented as $\delta_{i}(t)=0.1 \sin (3 t) \exp \left(-(t-5-0.2 i)^{2} / 4\right), \quad i=1,2, \ldots, 9$. The initial states of $\hat{\bar{\delta}}_{i}(0)$ and $\widehat{\widehat{\delta}}_{i}(0)$ are given as $\widehat{\bar{\delta}}_{i}(0)=0.1 *$ $[1,1,1,1,1,1,1,1,1]$ and $\widehat{\delta}_{i}(0)=-0.1 *[1,1,1,1,1,1,1,1,1]$ respectively. The other parameters for the proposed controller are set as $k=10, q=0.9, \gamma_{i}=0.02, \alpha_{1}=2, \alpha_{2}=1$, $\sigma=0.02, a=10$, and $b=0.0001$. On this basis, the simulation results of the proposed vehicle platoon control scheme are shown in Figure 3.

Figure 3 illustrates the trajectories of vehicles in a given line $\mathscr{L}$ with optimal spacing policy. As shown in Figure 3, the initial positions of vehicles are randomly located in the given line. Then, by using the proposed control law in (31), the vehicles will track their optimal positions, and the intervehicle space between any two consecutive vehicles varies dynamically with the road-friction coefficient. In addition, we can find that there is no collision during this process, which also validates the effectiveness of our proposed approaches. The detailed analyses are provided in Figures 4-9.

The evolution of tracking errors between the vehicles and their optimal positions are presented in Figure 4. As shown in this figure, the tracking errors converge to zeros in finite time. One may notice that there is some chattering around the time $150 \mathrm{~s}$ and $300 \mathrm{~s}$ in this figure. It is caused by the changing of the road-friction function. Since the roadfriction function $\phi(q)$ is changing based on (32), the value of $\phi(q)$ is decreasing around the time 150s. In this scenario, the optimal point $C_{V_{i}}$ may have a big gap according to (4), which

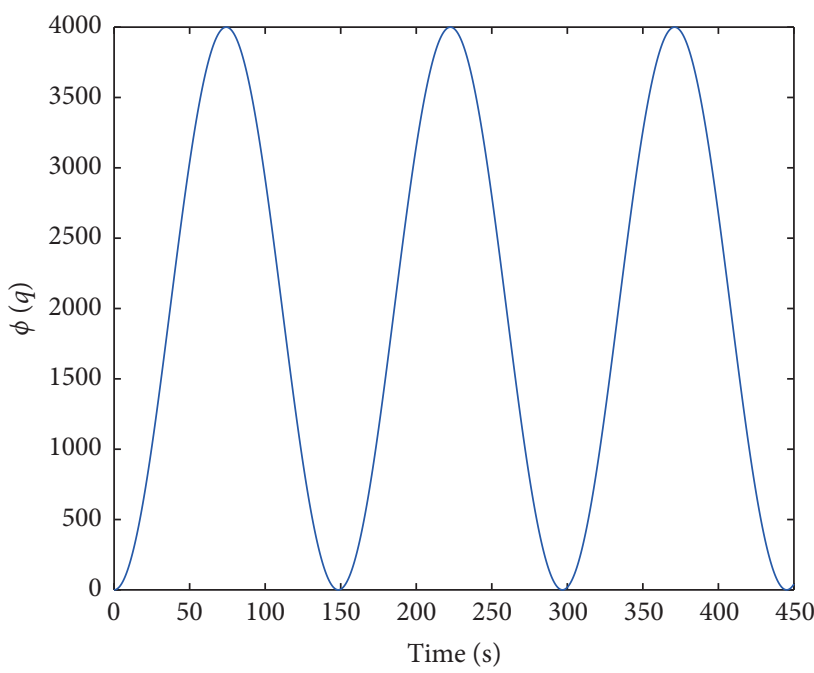

FIgURE 2: The evolution of road-friction coefficient on $\mathscr{L}$.

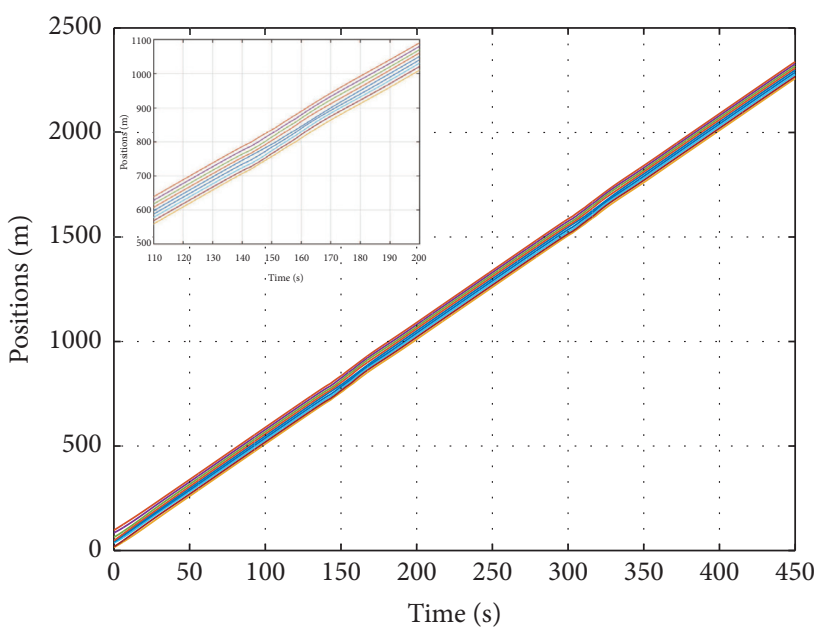

FIGURE 3: The optimal spacing platoon trajectories of vehicles. 


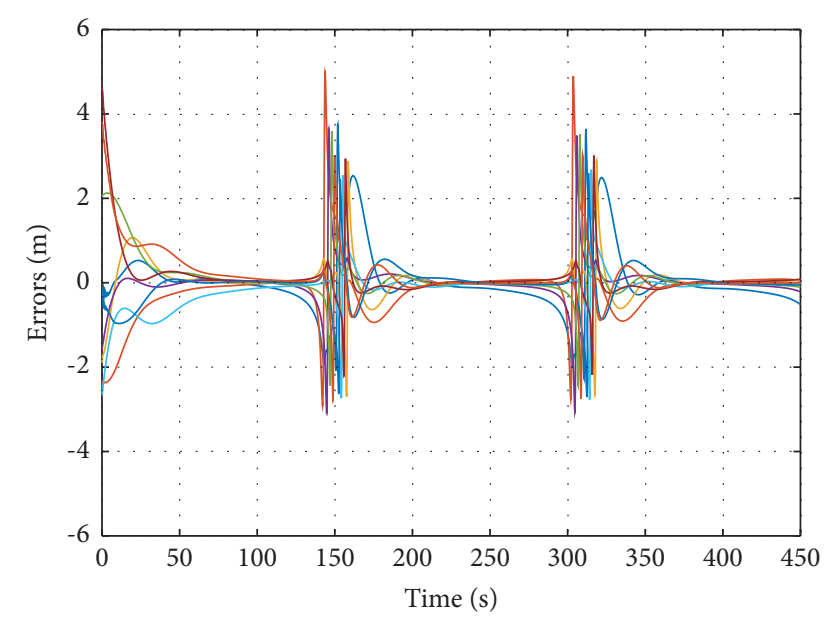

FIgURE 4: The tracing errors between the vehicles and their centroids.

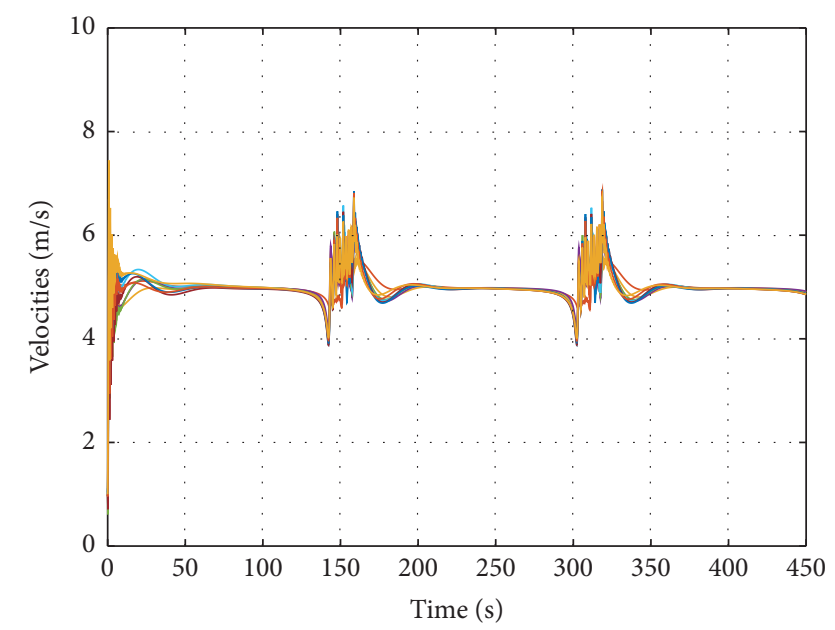

FIgUre 5: The evolution of all the vehicle velocities $v_{i}(t)$.

may lead a big chattering to the tracking errors. However, if one keeps using the proposed control scheme, the vehicle platoon will asymptotically converge to the zeros again. Hence, it shows that the proposed vehicle platoon control scheme is always feasible in various road-friction functions.

In Figure 5, the evolution of all the vehicle velocities is presented. As shown in Figure 5, all vehicles' velocities converge to the virtual leader. It shows that the vehicle platoon would effectively reach the desired platoon. Moreover, the reason of the chattering in this figure is the same with Figure 4. However, one can find that the vehicle will still converge to the leader after these chattering. It also shows the robustness of our proposed vehicle platoon control system.

The evolution of the sliding mode surface and the control inputs are presented in Figures 6 and 7, respectively. As we observed from these two figures, the sliding mode surface and the control inputs both converge to a constant. Particularly, for the chattering part, they would adjust

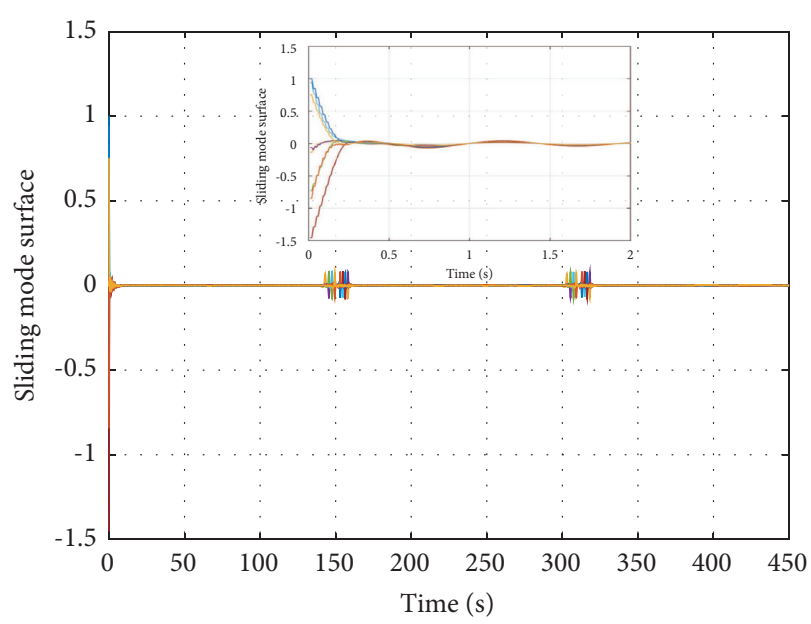

FIgURE 6: The evolution of the sliding mode surface $S_{i}(t)$.

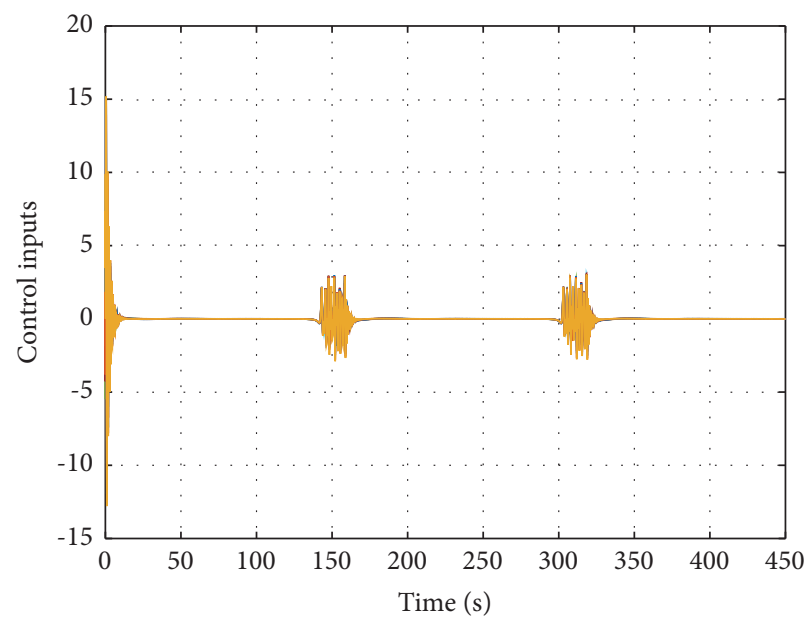

FIgURE 7: The evolution of the control inputs $u_{i}(t)$.

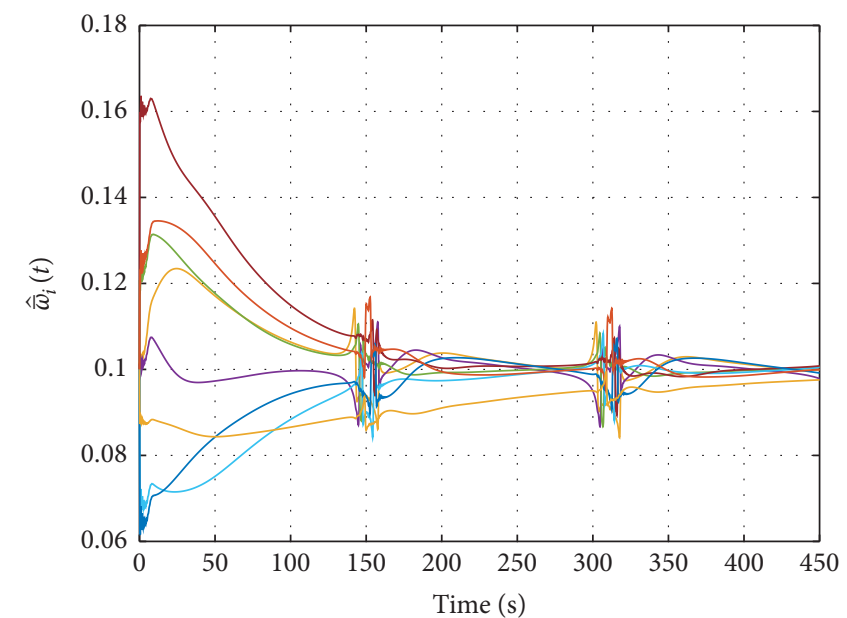

FIgURE 8: The estimation of upper bound disturbances $\widehat{\bar{\delta}}_{i}(t)$. 


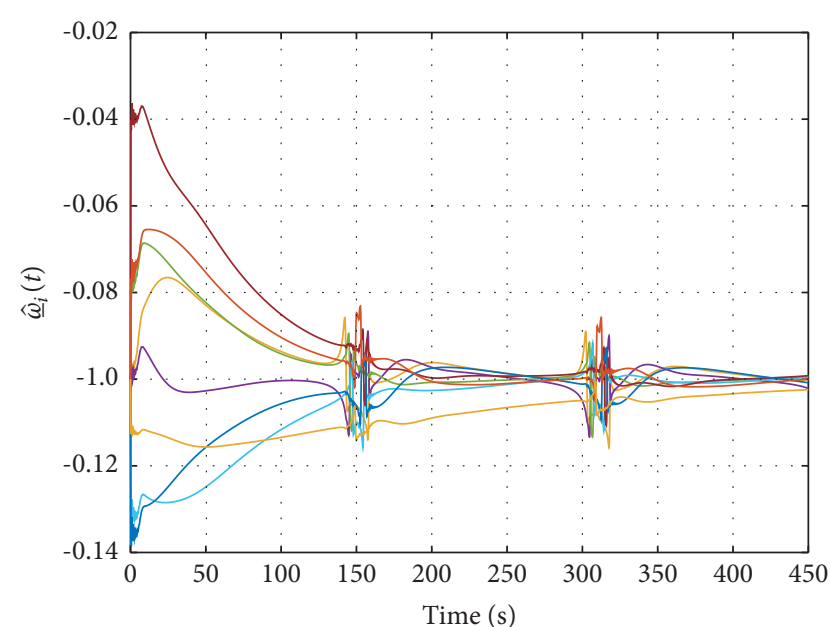

FIGURE 9: The estimation of lower bound disturbances $\widehat{\delta}_{i}(t)$.

themselves such that the vehicles can maintain the desired platoon.

In addition, for the external disturbances in the vehicle's dynamics, the estimations of its upper and lower bounds are shown in Figures 8 and 9, respectively. From these results, we can clearly find that the estimated boundaries will asymptotically converge to the constants. It shows the effectiveness of our proposed disturbance estimation methods in (21).

\section{Conclusion}

While considering the road-friction coefficient, we investigate the platoon control problems for a group of vehicles. In order to improve the driving safety of the vehicle platoon in various road conditions, an optimal spacing policy is proposed for the vehicle platoon with different road-friction coefficient. Then, an intervehicle space optimization framework is developed to find out the optimal spacing policy. This framework fully combines the vehicle platoon control and the road conditions, which would greatly improve vehicle platoon safety. Then, based on the proposed optimal spacing policy, a novel vehicle platoon control scheme is proposed by using the distributed integrated sliding mode (DISM). The external disturbances in the vehicle dynamics are also taken into consideration and a boundary estimation law is also developed for the vehicles. In final, the stability and feasibility of the proposed vehicle platoon control system with optimal spacing policy are strictly analyzed through theoretical derivation and numerical simulations.

Considering the vehicle safety and fuel economy, the optimal energy consumption model based on the research foundation of this paper will be proposed in the future, and then, a new platoon controller will be designed to achieve better control performance. We will try to adopt the method of cooperative adaptive cruise control (CACC) to solve these problems. Hence, we will investigate the optimal energy consumption policy for the vehicle platoon system with road-friction coefficient in the near future.

\section{Data Availability}

The data used to support the findings of this study are included within the article.

\section{Conflicts of Interest}

The authors declare that there are no conflicts of interest regarding the publication of this article.

\section{Acknowledgments}

This work was supported by the National Natural Science Foundation of China (nos. 51909008 and 61803040) and China Postdoctoral Science Foundation (no. 2021M692740).

\section{References}

[1] Y. Li, C. Tang, S. Peeta, and Y. Wang, "Nonlinear consensusbased connected vehicle platoon control incorporating carfollowing interactions and heterogeneous time delays," IEEE Transactions on Intelligent Transportation Systems, vol. 20, no. 6, pp. 2209-2219, 2019.

[2] M. Yan, W. Ma, L. Zuo, and P. Yang, "Dual-mode distributed model predictive control for platooning of connected vehicles with nonlinear dynamics," International Journal of Control, Automation \& Systems, vol. 17, no. 4, pp. 3901-3101, 2019.

[3] G. Guo, P. Li, and L.-Y. Hao, "Adaptive fault-tolerant control of platoons with guaranteed traffic flow stability," IEEE Transactions on Vehicular Technology, vol. 69, no. 7, pp. 6916-6927, 2020.

[4] G. Guo and Q. Wang, "Fuel-efficient en route speed planning and tracking control of truck platoons," IEEE Transactions on Intelligent Transportation Systems, vol. 20, no. 8, pp. 1-13, 2018.

[5] Y. Li, C. Tang, K. Li, X. He, S. Peeta, and Y. Wang, "Consensus-based cooperative control for multi-platoon under the connected vehicles environment," IEEE Transactions on Intelligent Transportation Systems, vol. 20, no. 6, pp. 2220-2229, 2019.

[6] J. Zhou and F. Zhu, "Analytical analysis of the effect of maximum platoon size of connected and automated vehicles," Transportation Research Part C: Emerging Technologies, vol. 122, Article ID 102882, 2021.

[7] B. Besselink and K. H. Johansson, "String stability and a delaybased spacing policy for vehicle platoons subject to disturbances," IEEE Transactions on Automatic Control, vol. 62, no. 9, pp. 4376-4391, 2017.

[8] A. Al Alam, A. Gattami, and K. H. Johansson, "An experimental study on the fuel reduction potential of heavy duty vehicle platooning," in Proceedings of the 13th International IEEE Conference on Intelligent Transportation Systems, pp. 306-311, IEEE, Funchal, Portugal, September 2010.

[9] R. Gabor and S. Zoltan, "Adaptation of spacing policy of autonomous vehicles based on an unknown input and state observer for a virtual predecessor vehicle," in Proceedings of the 2016 IEEE 55th Conference on Decision and Control (CDC), pp. 1715-1720, IEEE, Las Vegas, NV, USA, December 2016.

[10] J. Wang, X. Luo, W.-C. Wong, and X. Guan, "Specified-time vehicular platoon control with flexible safe distance constraint," IEEE Transactions on Vehicular Technology, vol. 68, no. 11, pp. 10489-10503, 2019. 
[11] T. Petrinić and I. Petrović, "Longitudinal spacing control of vehicles in a platoon for stable and increased traffic flow," in Proceedings of the 2012 IEEE International Conference on Control Applications, pp. 178-183, IEEE, Dubrovnik, Croatia, October 2012.

[12] L. Cheng and S. Panichpapiboon, "Effects of intervehicle spacing distributions on connectivity of vanet: a case study from measured highway traffic," IEEE Communications Magazine, vol. 50, no. 10, pp. 90-97, 2012.

[13] B. Pan and H. Wu, "Connectivity analysis in vehicular networks with slight traffic interference," Wireless Communications and Mobile Computing, vol. 2018, Article ID 9340239, 11 pages, 2018.

[14] R. G. Hoogendoorn, G. Tamminga, S. P. Hoogendoorn, and W. Daamen, "Longitudinal driving behavior under adverse weather conditions: adaptation effects, model performance and freeway capacity in case of fog," in Proceedings of the International IEEE Conference on Intelligent Transportation Systems, pp. 450-455, IEEE, Funchal, Portugal, September 2010.

[15] H. E. Sungu, M. Inoue, and J. Imura, "Nonlinear spacing policy based vehicle platoon control for local string stability and global traffic flow stability," in Proceedings of the 2015 European Control Conference (ECC), pp. 3396-3401, IEEE, Linz, Austria, July 2015.

[16] M. Yan, W. Ma, L. Zuo, and P. Yang, "Distributed model predictive control for platooning of heterogeneous vehicles with multiple constraints and communication delays," Journal of Advanced Transportation, vol. 2020, Article ID 4657584, 16 pages, 2020.

[17] Y. Zheng, S. Eben Li, J. Wang, D. Cao, and K. Li, "Stability and scalability of homogeneous vehicular platoon: study on the influence of information flow topologies," IEEE Transactions on Intelligent Transportation Systems, vol. 17, no. 1, pp. 14-26, 2016.

[18] G. Guo and D. Li, "Adaptive sliding mode control of vehicular platoons with prescribed tracking performance," IEEE Transactions on Vehicular Technology, vol. 68, no. 8, pp. 7511-7520, 2019.

[19] J. Hu, P. Bhowmick, F. Arvin, A. Lanzon, and B. Lennox, "Cooperative control of heterogeneous connected vehicle platoons: an adaptive leader-following approach," IEEE Robotics and Automation Letters, vol. 5, no. 2, pp. 977-984, 2020.

[20] S. Wen and G. Guo, "Control of leader-following vehicle platoons with varied communication range," IEEE Transactions on Intelligent Vehicles, vol. 5, no. 2, pp. 240-250, 2020.

[21] C. Zhai, F. Luo, and Y. Liu, "Cooperative power split optimization for a group of intelligent electric vehicles travelling on a highway with varying slopes," IEEE Transactions on Intelligent Transportation Systems, pp. 1-13, 2020.

[22] C. Zhai, X. Chen, C. Yan, Y. Liu, and H. Li, "Ecological cooperative adaptive cruise control for a heterogeneous platoon of heavy-duty vehicles with time delays," IEEE Access, vol. 8, pp. 146208-146219, 2019.

[23] X. Guo, J. Wang, F. Liao, and R. S. H. Teo, "Distributed adaptive integrated-sliding-mode controller synthesis for string stability of vehicle platoons," IEEE Transactions on Intelligent Transportation Systems, vol. 17, no. 9, pp. 24192429, 2016.

[24] B. Bayar, S. A. Sajadi-Alamdari, F. Viti, and H. Voos, "Impact of different spacing policies for adaptive cruise control on traffic and energy consumption of electric vehicles," in Proceedings of the 2016 24th Mediterranean Conference on Control and Automation (MED), pp. 1349-1354, IEEE, Athens, Greece, June 2016.

[25] G. Rodonyi, "An adaptive spacing policy guaranteeing string stability in multi-brand ad hoc platoons," IEEE Transactions on Intelligent Transportation Systems, vol. 19, no. 6, pp. 1902-1912, 2018.

[26] Y. Li, C. Tang, S. Peeta, and Y. Wang, "Integral-sliding-mode braking control for a connected vehicle platoon: theory and application," IEEE Transactions on Industrial Electronics, vol. 66, no. 6, pp. 4618-4628, 2019.

[27] Y. Ma, Z. Li, R. Malekian, R. Zhang, X. Song, and M. A. Sotelo, "Hierarchical fuzzy logic-based variable structure control for vehicles platooning," IEEE Transactions on Intelligent Transportation Systems, vol. 20, no. 4, pp. 1329-1340, 2018.

[28] D. Li and G. Guo, "Prescribed performance concurrent control of connected vehicles with nonlinear third-order dynamics," IEEE Transactions on Vehicular Technology, vol. 69, no. 12, pp. 14793-14802, 2020.

[29] C. Zhai, Y. Liu, and F. Luo, "A switched control strategy of heterogeneous vehicle platoon for multiple objectives with state constraints," IEEE Transactions on Intelligent Transportation Systems, vol. 20, no. 5, pp. 1883-1896, 2019.

[30] K. Shojaei and M. R. Yousefi, "Tracking control of a convoy of autonomous robotic cars with a prescribed performance," Transactions of the Institute of Measurement and Control, vol. 41, no. 10, pp. 3725-3741, 2019.

[31] K. Shojaei and A. Chatraei, "Robust platoon control of underactuated autonomous underwater vehicles subjected to nonlinearities, uncertainties and range and angle constraints," Applied Ocean Research, vol. 110, no. 6, pp. 1-15, 2021.

[32] O. Elhaki and K. Shojaei, "Observer-based neural adaptive control of a platoon of autonomous tractor-trailer vehicles with uncertain dynamics," IET Control Theory \& Applications, vol. 14, no. 14, pp. 1898-1911, 2020.

[33] Q. Du, V. Faber, and M. Gunzburger, "Centroidal voronoi tessellations: applications and algorithms," SIAM Review, vol. 41, no. 4, pp. 637-676.

[34] H. K. Khalil, "Output regulation of uncertain nonlinear systems," Automatica, vol. 38, no. 6, pp. 1091-1093, 2002. 